\title{
Two bolometer arrays for far-infrared and submillimeter astronomy
}

Robert F. Silverberg, Christine A. Allen, Sachidananda R. Babu, Dominic J. Benford, David T. Chuss, et al.

Robert F. Silverberg, Christine A. Allen, Sachidananda R. Babu, Dominic J. Benford, David T. Chuss, Jessie L. Dotson, Charles Darren Dowell, Doyle A. Harper, Murzy D. Jhabvala, Robert F. Loewenstein, S. Harvey Moseley, Johannes G. Staguhn, George M. Voellmer, Edward J. Wollack, "Two bolometer arrays for far-infrared and submillimeter astronomy," Proc. SPIE 5498, Millimeter and Submillimeter Detectors for Astronomy II, (8 October 2004); doi: 10.1117/12.552422

Event: SPIE Astronomical Telescopes + Instrumentation, 2004, Glasgow, United Kingdom 


\title{
Two Bolometer Arrays for Far-Infrared and Submillimeter Astronomy
}

\author{
R. F. Silverberg ${ }^{a}$, C. A. Allen ${ }^{a}$, S. R. Babu ${ }^{a}$, D. J. Benford ${ }^{a}$, D. T. Chuss ${ }^{a}$, J. L. Dotson $^{b}$, \\ C. D. Dowell ${ }^{c, d}$, D. A. Harper ${ }^{e}$, M. D. Jhabvala ${ }^{a}$, R. F. Loewenstein ${ }^{e}$, S. H. Moseley ${ }^{a}$, \\ J. G. Staguhn ${ }^{a}$, G. Voellmer ${ }^{a}$, E. J. Wollack ${ }^{a}$ \\ ${ }^{a}$ NASA/Goddard Space Flight Center, Greenbelt, MD 20771 USA \\ ${ }^{b}$ NASA Ames Research Center, Moffet Field, CA 94035 \\ ${ }^{c}$ NASA/JPL, Pasadena, CA 91109 USA \\ ${ }^{d}$ California Institute of Technology, Pasadena, CA 91125 USA \\ eYerkes Observatory, University of Chicago, Williams Bay, WI 53191 USA
}

\begin{abstract}
We describe the development, construction, and testing of two 384 element arrays of ion-implanted semiconducting cryogenic bolometers designed for use in far-infrared and submillimeter cameras. These two dimensional arrays are assembled from a number of 32 element linear arrays of monolithic Pop-Up bolometer Detectors (PUD) developed at NASA/Goddard Space Flight Center. PUD technology allows the construction of large, high filling factor, arrays that make efficient use of available focal plane area in far-infrared and submillimeter astronomical instruments. Such arrays can be used to provide a significant increase in mapping speed over smaller arrays. A prototype array has been delivered and integrated into a ground-based camera, the Submillimeter High Angular Resolution Camera (SHARC II), a facility instrument at the Caltech Submillimeter Observatory (CSO). A second array has recently been delivered for integration into the High-resolution Airborne Widebandwidth Camera (HAWC), a far-infrared imaging camera for the Stratospheric Observatory for Infrared Astronomy (SOFIA). HAWC is scheduled for commissioning in 2005.
\end{abstract}

Keywords: bolometer, far-infrared, sub-millimeter, detector arrays, instrumentation, CSO, SOFIA

\section{INTRODUCTION}

Recent advances in submillimeter and far-infrared detector technology have made it possible to produce large arrays of background-limited detectors. Several groups have taken advantage of the first generation of these arrays to provide improved mapping speed to study the population of high redshift galaxies, the Sunyaev-Zel'dovich effect, and the Cosmic Background Radiation (CMBR) from a variety of platforms. A number of the instruments using array technology use conical feedhorns to concentrate the light from large areas of the focal plane onto the smaller area of the bolometric detector. An alternate approach, filling all or a significant portion of the available focal plane with a two dimensional filled array of bare detectors, provides faster mapping speeds than feedhorn-coupled implementations.

The development of Pop-up Detector (PUD) ${ }^{1}$ technology at NASA/Goddard Space Flight Center has allowed us to implement bare filled array focal planes in two instruments. PUDs provide several advantages over some earlier bolometer architectures. Nearly filled arrays are possible. PUDs achieve filling factors $>95 \%$ because the electrical/thermal leads are folded out of the focal plane. In addition, depending on the bandwidth requirements, it is possible to implement absorber strategies that achieve absorption efficiencies $>90 \%$ over a finite bandwidth. In this paper, we describe the development of two $12 \times 32$ PUD arrays. The first array, a prototype for the HAWC array, is now successfully operating in the SHARC $\mathrm{II}^{2}$ camera, a facility instrument at the Caltech Submillimeter Observatory (CSO). SHARC II was commissioned in 2002. The second array has only recently been delivered to

Further author information: (Send correspondence to: R. F. S. )

E-mail: Robert.Silverberg@nasa.gov, Telephone: 13012867468 
the University of Chicago, Yerkes Observatory for integration into HAWC, ${ }^{3}$ a facility imaging photometer for NASA's Stratospheric Observatory for Infrared Astronomy(SOFIA).

In the next sections, we briefly describe the two instruments and their characteristics. In sections 4 and 5 , we review the pop-up technology and array construction and in section 6 we discuss some differences in how the arrays for the two different instruments are implemented. Section 7 describes the testing and construction of the most recent array for HAWC. Finally, we discuss the current status of the arrays and further work that is planned.

\section{SHARC II}

In contrast to many earlier ground-based and balloon-borne bolometer arrays, SHARC II uses a filled "bare" two dimensional bolometer array to provide full Nyquist sampling for each field of view. This provides optimal mapping speeds when compared to horn-fed arrays ${ }^{4,5}$, but may require some care to avoid significant loading of the detectors from radiation within the cryostat itself. However, for broadband ground-based applications at submillimeter wavelengths, the loading from the sky will often far exceed the internal loading from the cryostat, so extraordinary measures to reduce cryostat loading are not required.

The prototype array for the HAWC development effort was used in SHARC II. SHARC II is optimized for operation in the 350 and $450 \mu \mathrm{m}$ atmospheric windows; however, it is also capable of undertaking observations at $850 \mu \mathrm{m}$. The $10 \mathrm{~m}$ aperture of the CSO provides an angular resolution of $9^{\prime \prime}$ at $350 \mu \mathrm{m}$ and the array pixels are $0.65 \lambda / D$ at that wavelength. Scientifically, these observations will serve to complement future HAWC observations by providing long wavelength data at comparable angular resolution. SHARC II has demonstrated near background-limited performance under all weather conditions where observations are practical.

\section{HAWC}

Offering a larger primary mirror than contemporary satellites operating at comparable wavelengths, SOFIA will achieve spatial resolutions in the far-infrared that will allow the most detailed maps of far-infrared sources to date. HAWC's role is challenging. It will be used to address many scientific questions. These include studies of star formation and stellar clusters in our Galaxy, star formation in external galaxies, structure and evolution of protoplanetary disks, composition and the life-cycle of interstellar dust. Far-Infrared observations with high angular resolution are required for these problems and many of HAWC's observations will complement observations from instruments such as SHARC II and the suite of instruments aboard NASA's space-based infrared observatory, the Spitzer Space Telescope.

As the facility imaging camera for SOFIA, HAWC will provide high resolution mapping and photometric capability to SOFIA. It will have four passbands from $40 \mu \mathrm{m}$ through $300 \mu \mathrm{m}$ and therefore will be able to measure spectral energy distributions (SEDs) of sources at high angular resolution. The four HAWC nominal band centers are given in Table 1 along with their bandwidths and angular resolutions. The fractional wavelength range for HAWC is much broader than that of SHARC II. As such the HAWC array is optimized somewhat differently. The array maintains the $12 \times 32$ format of $1 \mathrm{~mm} \times 1 \mathrm{~mm} \times 1 \mu \mathrm{m}$ pixels but it is designed for operation at a lower bath temperature $(200 \mathrm{mK})$. The ion-implantation dose is somewhat higher to achieve the desired resistance at the lower operating temperature.

\section{POP-UP DETECTORS}

Both the HAWC and SHARC II PUD detectors are fabricated on a $1 \mu \mathrm{m}$ silicon membrane. The entire $1 \mathrm{~mm} \mathrm{x}$ $1 \mathrm{~mm}$ area of the detector is ion implanted with boron and phosphorus to form the semiconducting thermistor. This large thermistor reduces the $1 / \mathrm{f}$ noise knee frequency in the bolometer. The linear arrays are folded on a ceramic busbar with their surfaces precisely spaced above the busbars. Two-dimensional arrays of bolometers are constructed from constituent $1 \times 32$ linear arrays. ${ }^{6} \quad$ A segment of a folded linear array is shown in Figure 2 . Each linear array consists of $321 \mathrm{~mm} \times 1 \mathrm{~mm}$ bolometer membranes that are fabricated from a single piece of silicon. Silicon straps on either side of each bolometer membrane provide support for the pixels to the frame. Aluminum traces run down both straps to provide both thermal and electrical connections to the frame. Figure 1 shows a schematic of a small section of a $1 \times 32$ linear array before it has been folded. 
Table 1. HAWC Parameters

\begin{tabular}{|c|c|c|}
\hline$\lambda$ & $\Delta \lambda / \lambda$ & Angular \\
\hline$(\mu \mathrm{m})$ & & Resolution(" $\left.{ }^{\prime \prime}\right)$ \\
\hline 53 & 0.16 & 6 \\
\hline 89 & 0.19 & 9 \\
\hline 155 & 0.22 & 15 \\
\hline 216 & 0.21 & 21 \\
\hline
\end{tabular}

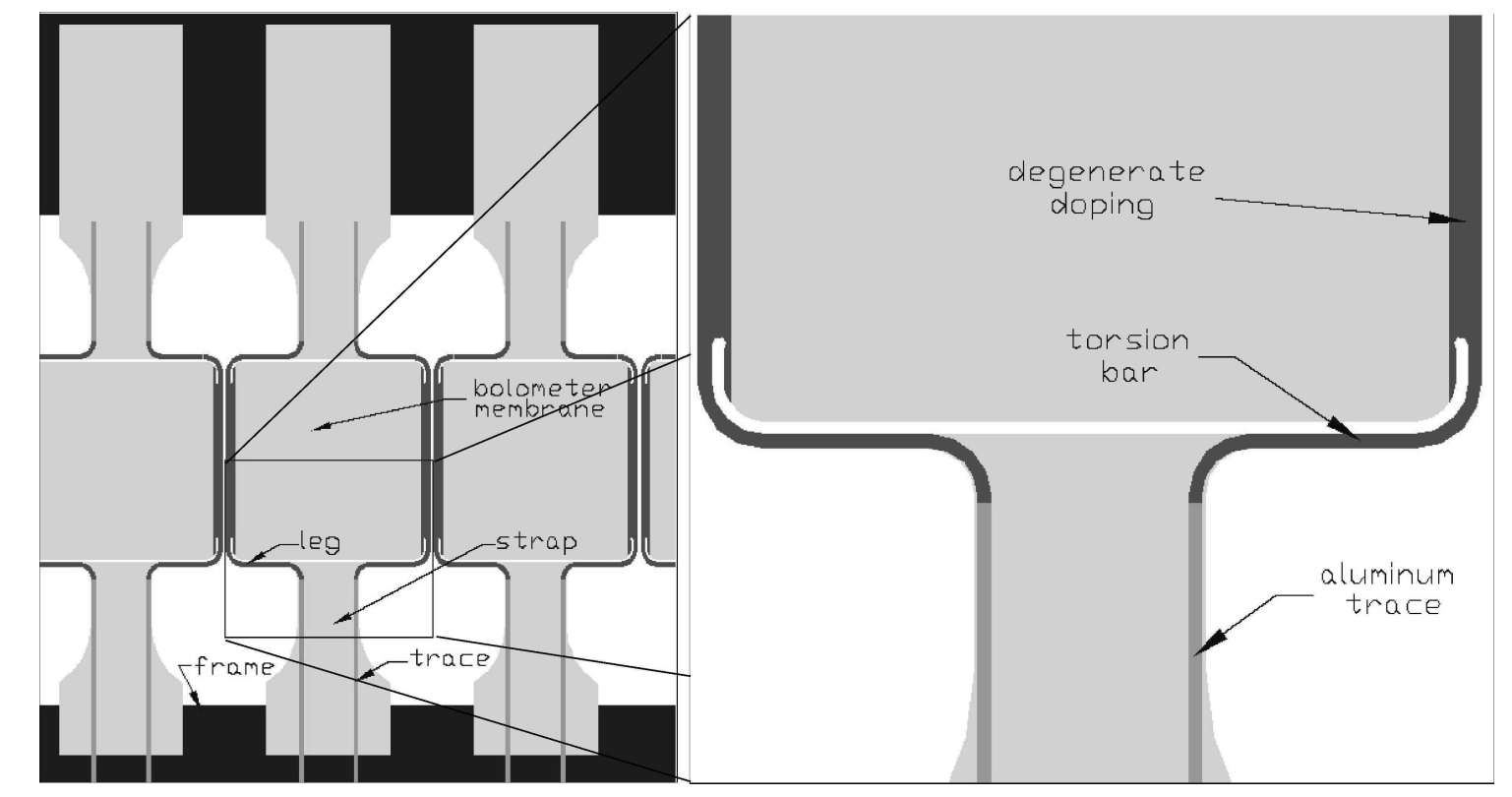

Figure 1. A schematic of a section of the unfolded $1 \times 32$ linear array is shown. ${ }^{2}$ The blowup of the inset region on the right shows the details of the torsion bar that allows the straps to be folded out of the image plane. 


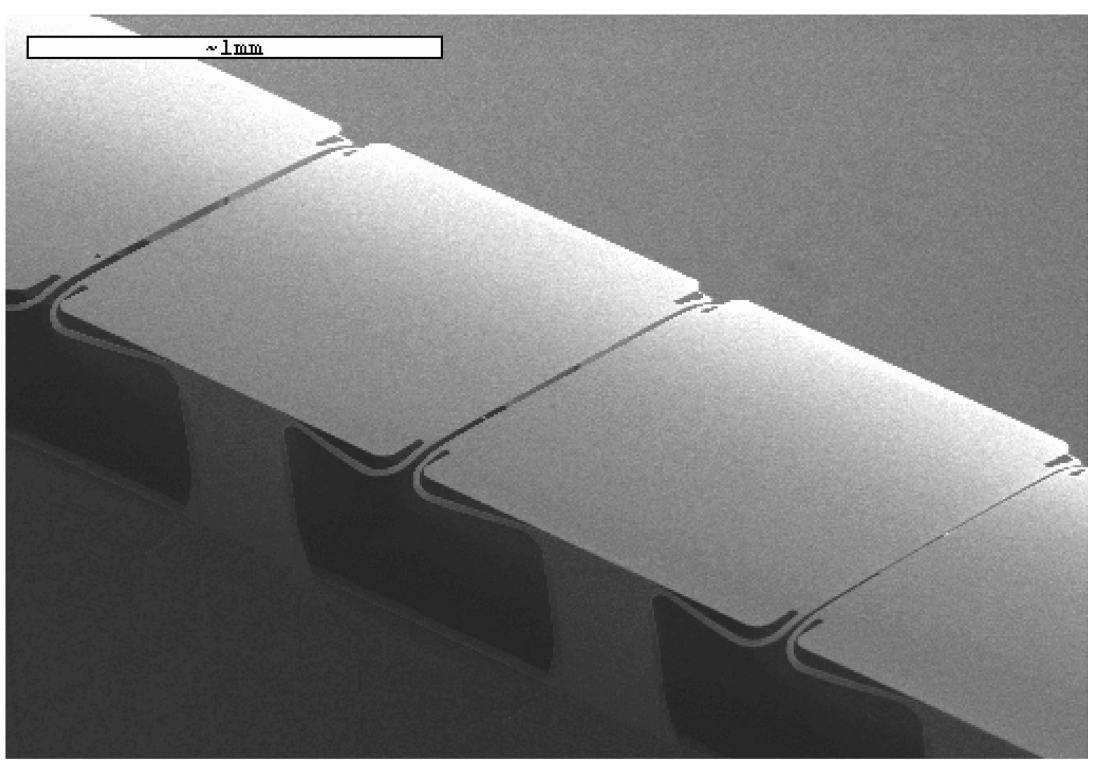

Figure 2. A photograph of a section of the folded $1 \times 32$ linear array. Most of the stress in the silicon is taken by the torsion bars seen going below the image plane.

The individual linear arrays are deposited with an appropriate absorber coating and then the frames are folded back onto the sides of a ceramic busbar. The stress of the fold is absorbed by the torsion bars on either side of the bolometer membrane (see Figure 2). In this way, the area to either side of the bolometer membranes is unobstructed and thus free to accommodate adjacent rows of PUDs. This construction allows the close packaging of the linear arrays to form a filled, close-packed, two dimensional array.

\section{THERMAL ISOLATION AND FRONT END ELECTRONICS}

To read out the array, each bolometer is operated nearly current-biased. A large value, custom-made, load resistor $(\sim 130 \mathrm{M} \Omega$ for SHARC II) is placed on the readout drawer where each linear bolometer array is mounted. The load resistors are heat sunk to the liquid helium bath and are thermally isolated from the lower temperature detectors using a microbridge thermal isolator. ${ }^{6}$ This same device is used to isolate the the liquid helium bath from the JFET preamplifier operating at an elevated temperature.

Since there is not a practical, low-power method of multiplexing semiconducting bolometers, both SHARC II and HAWC use a JFET source follower amplifier for each bolometer in the array. The source follower sends the bolometer signal to an external amplifier. The 384 JFETs used to implement these front-end amplifiers are thermally-isolated and radiatively shielded from the bolometer array. The internal JFETs operate at $\sim 120 \mathrm{~K}$ for minimum voltage noise. The power requirement for these JFETs and the thermal conduction of the large number of wires sets a limit to the size of the array that is practical in a many applications. The $12 \times 32$ array of semiconducting bolometers, the load resistors, and thermally-isolated JFETs are packaged in a single structure heat sunk the $4 \mathrm{~K}$ bath of the cryostat. The detector array is separately heat sunk to the $300 \mathrm{mK}{ }^{3} \mathrm{He}$ refrigerator stage for SHARC II or to the $200 \mathrm{mK}$ single-stage Adiabatic Demagnetization Refrigerator for HAWC . A detailed description of the mechanical and thermal arrangement for SHARC II can be found in Reference 6 .

\section{ABSORBER STRATEGIES}

Absorber strategies for the HAWC and SHARC II arrays differ. For SHARC II, the close spectral proximity of the two primary atmospheric windows at 350 and $450 \mu \mathrm{m}$ allows the efficient implementation of a resonant absorber that works well across both bands. The absorbing strategy of SHARC II consists of a $\sim 400 \Omega / \square$ 
coating of bismuth absorber on the bolometer membrane accompanied by a quarter wave backshort located 100 $\mu \mathrm{m}$ behind the pixels. This configuration allows for $>95 \%$ absorption across these two SHARC II bands. The transmission line model and a schematic representation of this strategy are shown in the left side of Figure 3. A backshort is formed by coating the surface of the ceramic busbar where the bolometers are mounted with gold. In practice, the absorption is dependent on the precision of backshort placement and surface impedance of the absorbing film on the bolometer.

For HAWC, the spectral range of its four bands is much larger, spanning nearly a decade in wavelength coverage. It would be difficult to properly match these windows at all bands with a single resonant backshort. The HAWC absorber strategy was chosen to maximize the bandwidth of the absorber. As shown in the right hand side of Figure 3, the bolometer membrane is coated with an optimal surface impedance of $157 \Omega / \square$. Bismuth has an unacceptable reactance over parts of the HAWC passband, so a different material had to be chosen for the coating of the HAWC bolometers. The absorbing layer is a thin film metal bilayer of titanium-gold (Ti-Au). The gold-plated busbar on which the linear arrays are mounted was painted with an epoxy infused with carbon lamp black and $40 \mu \mathrm{m}$ glass beads to form a cold absorber. This leads to a predicted $50 \%$ absorption that is uniform across all four passbands. Actual detector performance will depend on the values of the surface impedances of the Ti-Au films and the efficiency of the busbar absorber.

During tests of the Ti-Au coatings, they were found to be slightly unstable when stored at room temperatures for periods of a year or longer (accelerated aging tests were performed). The instability manifests itself in a slow rise of the surface impedance leading to reduced absorption as the devices age. Thus, to maintain the HAWC array it will be stored below $-40^{\circ} \mathrm{C}$ when not in use. This cold storage has been found to arrest the aging process.

\section{HAWC SUB-ARRAY TESTING}

The fundamental unit in the construction of the two dimensional HAWC array is the folded, absorber-coated linear array, referred to as a row. To provide high uniformity across the arrays, many PUD linear arrays are fabricated. They then undergo a series of electrical and optical tests at cryogenic temperatures that define the criteria for downselecting the candidates to the 12 rows that make up the 384 pixel HAWC array. The rows were tested, two at a time, in a ${ }^{3} \mathrm{He}$-cooled test dewar. The procedures for testing the the linear arrays were similar for those that were performed for SHARC $\mathrm{II}^{7}$ and verify the bolometer parameters: thermal conductivity, resistance at the operating temperature, dark noise, and detector time constant.

Load curves are taken at three bath temperatures: 250, 300, and $450 \mathrm{mK}$. The current-voltage response of the detectors are found by changing the bias voltage across the load resistor/bolometer combination and measuring the DC voltage across the bolometer. A sample load curve is shown in Figure 4A. With this data at the different temperatures, one can estimate the properties of the bolometer by fitting the load curves to eqns (1). The resistance, $R$, and thermal conductivity, $G$ of the bolometers can be modelled by the equations

$$
R=R_{o} \exp \sqrt{T_{o} / T}, G=G_{o} T^{\beta}
$$

The estimations of $R_{o}, T_{o}, G_{o}$, and $\beta$ allow for selection of linear arrays and optimal physical placement of the bolometers in the final two-dimensional configuration. In the HAWC cryostat, each adjacent pair of bolometers shares a bias voltage. Thus, by placing electrically similar rows next to one another, the six independent bias lines can be adjusted to maximize the uniformity of response of the array.

The time constants of the bolometers on each row are estimated. This is done by adding a small amplitude, slow square wave signal to the bias voltage of the row under test in the dark cryostat at the desired test temperature and reading out the bolometers with a unity gain DC amplifier. The DC-coupled amplifier allows us to measure the properties of the bolometer without rolloff from the amplifier. At each transition in the square wave, the bolometer receives an impulse and then nearly exponentially recovers to a new "steady-state" condition. As long as the square wave amplitude is very small, this approximates the bolometer's response to a an impulsive radiative signal. Because of the small amplitude of the square wave, it is difficult to determine the decay time from a single transition in the square wave. The responses of the detectors over many cycles of this signal are synchronously co-added, and a fit is done to the co-added data to determine the bolometer's time 
SHARC II
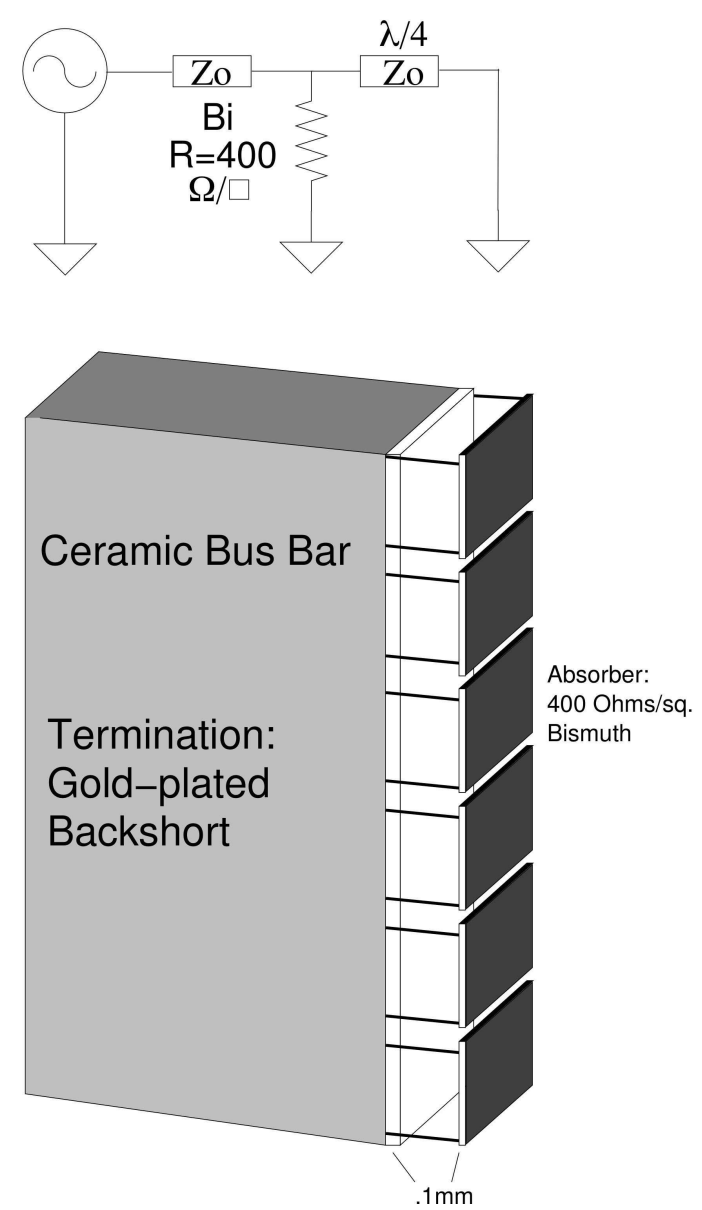

\section{HAWC}
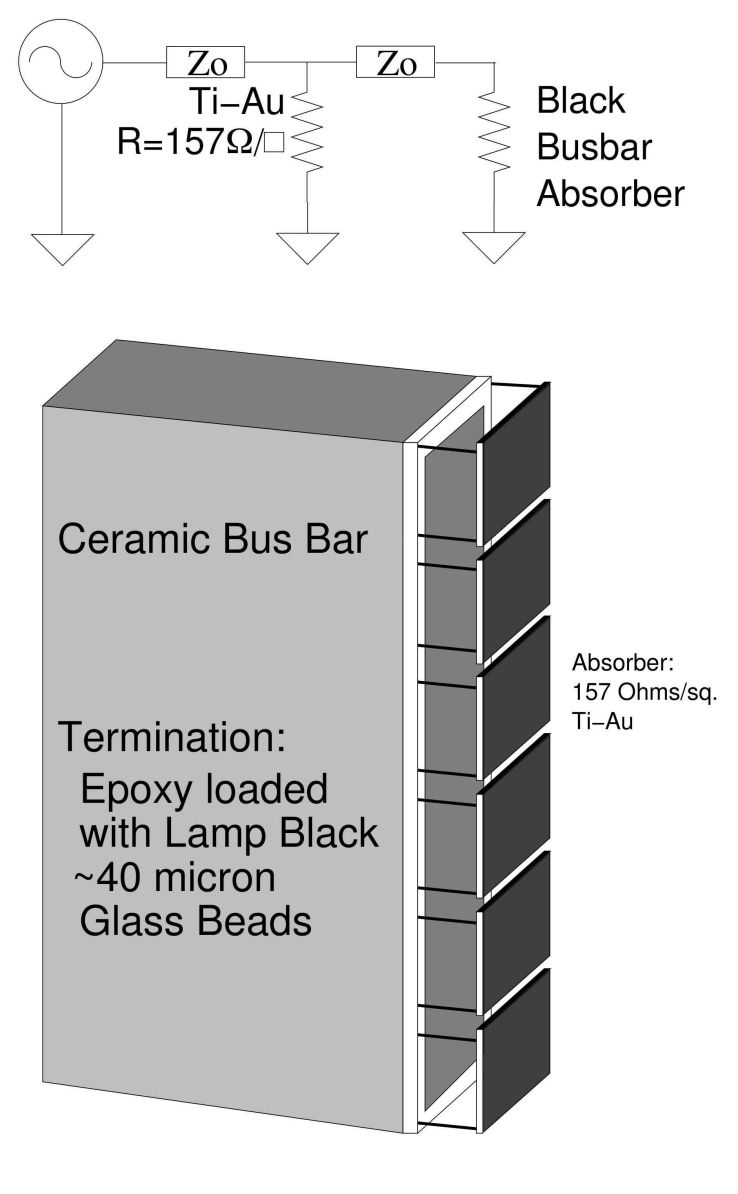

Figure 3. Contrasting absorber strategies for SHARC II and HAWC are shown along with their transmission line representation. For HAWC, the absorption scheme is such as to allow for 50\% absorption over the entire $40-300 \mu \mathrm{m}$ HAWC band. The SHARC II strategy is optimized for $\sim 95 \%$ absorption over the 350 and $450 \mu \mathrm{m}$ bands. 
constant. For the HAWC detector, the time constants for each detector must be below 10 ms. Figure $4 \mathrm{~B}$ shows a typical time constant data set with an exponential fit to the data.

Noise tests are done on each row in order to check for rows or individual devices that may have excess noise. The rows are placed in the test dewar in the dark. The detectors are read out with a high gain AC amplifier so the low noise from the bolometers can be amplified and digitized. The power spectrum of the digitized data stream is then computed to produce the dark noise spectrum of the device under test. In these tests, the detectors were DC biased so noise below $1 \mathrm{~Hz}$ is enhanced by the amplifier. In the case of SHARC II, an AC biasing and demodulation scheme at $100 \mathrm{~Hz}$ is used and the noise spectrum of the amplifier and detector is nearly flat down to $\sim 100 \mathrm{mHz}$. When testing the HAWC/SHARC II devices in the dark test cryostat, baseline noise levels were found to be $20-40 \mathrm{nV} / \sqrt{\mathrm{Hz}}$. With typical responsivities of $3-4 \times 10^{8} \mathrm{~V} / \mathrm{W}$, detector NEPs of $7-13 \times 10^{-17}$ $\mathrm{W} / \sqrt{H} z$ are estimated. The background-limited NEPs for HAWC are expected to be $2.2-6.6 \times 10^{-16} \mathrm{~W} / \sqrt{H} z,^{3}$ so these detectors should achieve near background-limited performance under nominal observing conditions.

Radiative tests were also done on the HAWC arrays using a pulsed, unfocused, and uncalibrated reverse bolometer ${ }^{8}$ behind a $350 \mu \mathrm{m}$ filter. This source verified that submillimeter radiation coupled to the bolometers and that the PUD to PUD characteristics were consistent. In addition, it provided an additional check on the consistency of the absorber coatings from linear array to linear array. Only a subset of the HAWC linear arrays were tested in this way.
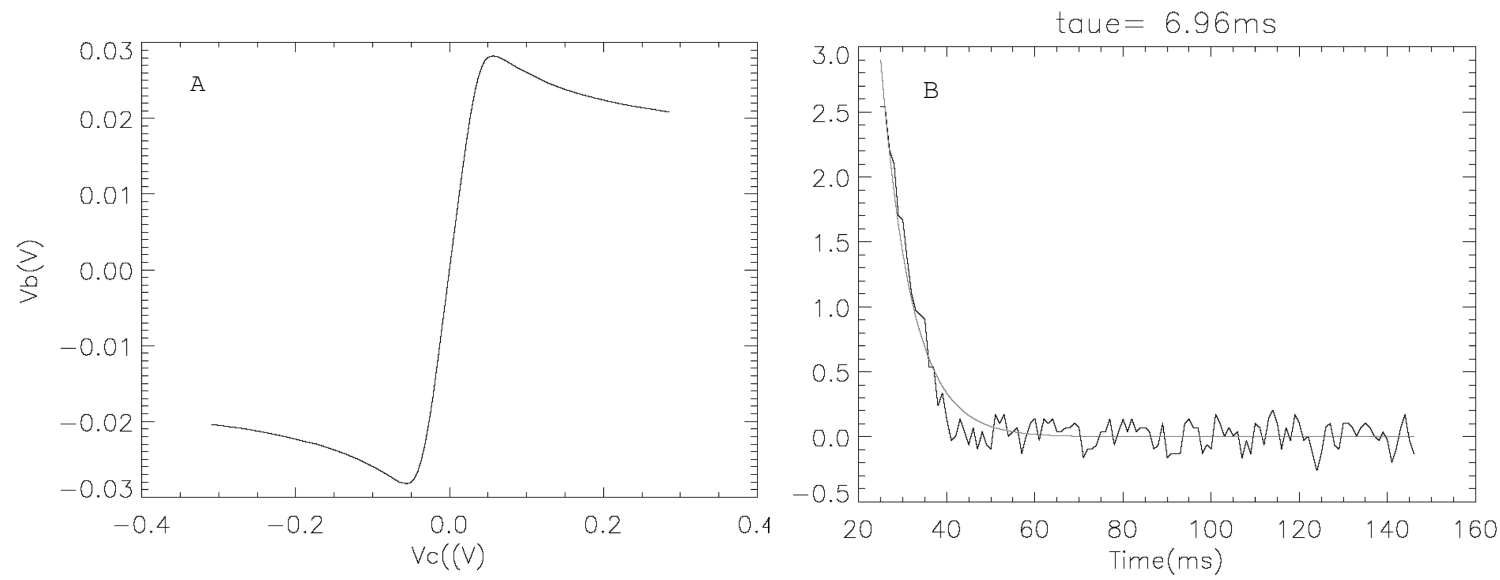

Figure 4. Characterization of individual PUDs. (A) The plot on the left shows a typical load curve. The horizontal axis is the voltage across the load resistor and bolometer. Since the load resistor is much larger than the bolometer resistance, this voltage is nearly proportional to the bias current. The vertical axis is the voltage across the bolometer itself. (B) The plot on the right shows a set of synchronously coadded time constant data (jagged line). The smooth line is the best fit to an exponential decay.

In addition to the parametric selection of matching characteristics, two other selection criteria were considered in choosing the final linear arrays. The first was that there be no more than $10 \%$ of the pixels that were bad, either because they were broken, inoperative or excessively noisy. Therefore, linear arrays containing more than 3 bad pixels were set aside. The second criteria added was that rows having lower surface impedances were given higher rankings than those with higher surface impedances because those are expected to age more slowly than rows having higher surface impedance..

\section{CURRENT STATUS AND FUTURE PLANS}

The SHARC II is currently available for observers at the CSO with a $2.5^{\prime} \times 0.9^{\prime}$ field of view at $350 \mu \mathrm{m}$. A scanning mode without chopping has been successfully used for data aquisition. Sensitivity of $\sim 1 \mathrm{Jy} \sqrt{s}$ at an optical depth, $\tau_{225 G H z}=0.05$ was achieved in January 2003. Figure 5 shows an example of the high quality 


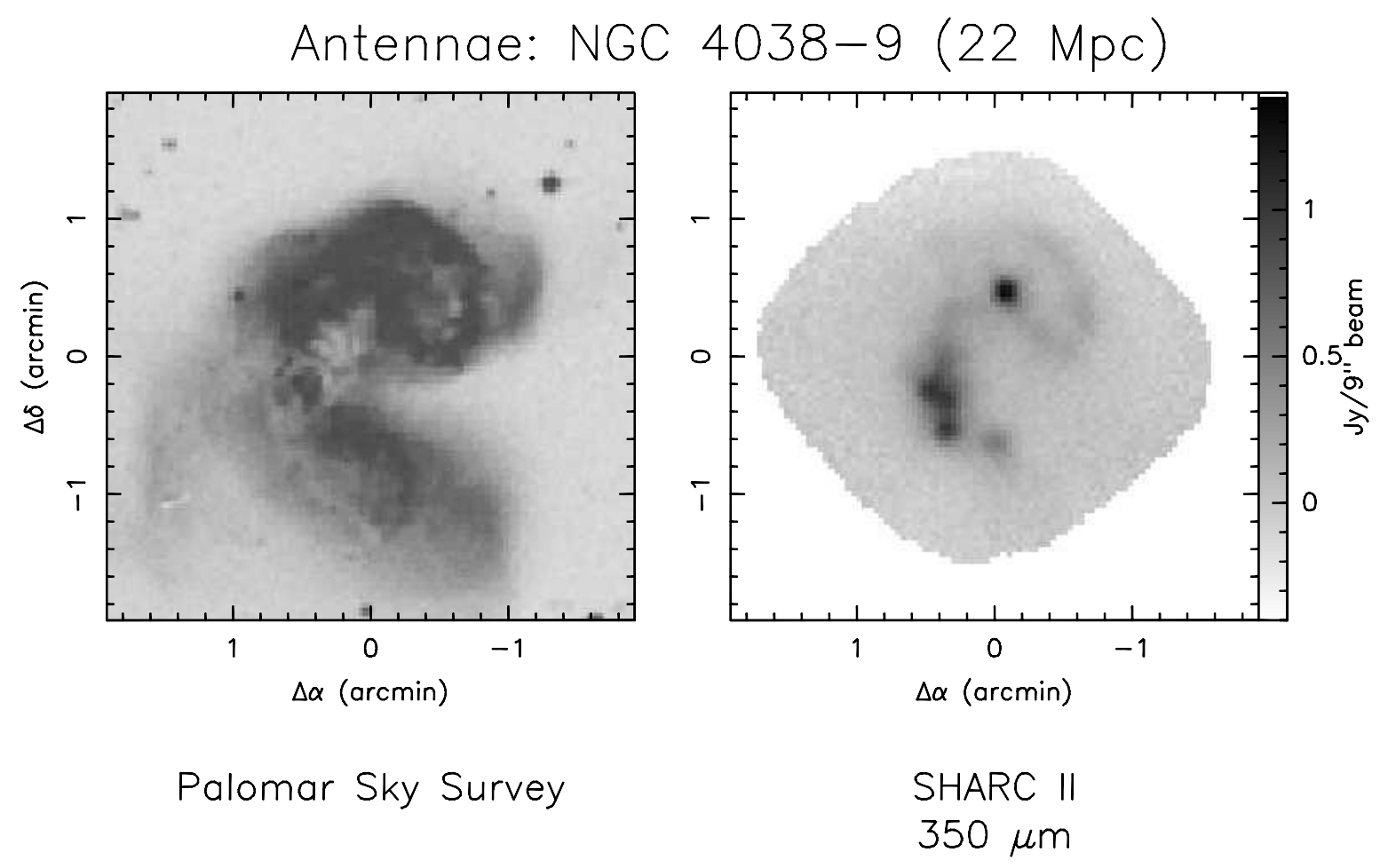

Figure 5. Antennae Nebulae at $350 \mu$ m observed with SHARC II in 2003 compared to the optical image. ${ }^{9}$ The merging galaxies are easily seen at this resolution. The noise level in the $350 \mu \mathrm{m}$ map is about $20 \mathrm{mJy}$.

imaging capabilities of this instrument. The HAWC array has been completed and is being prepared for a testing phase and integration into the instrument. First light for HAWC is scheduled for a SOFIA flight in 2005.

There are no plans for additional PUD arrays of ion-implanted semiconducting bolometers, but some arrays of PUDs with superconductingTransition Edge Sensors (TES) as the temperature-sensing element are being developed for use on ground-based ${ }^{10}$ and airborne ${ }^{11,12}$ instruments. The bolometers with TES thermistors can be multiplexed using devices such as the low power SQUID multiplexers developed at NIST. ${ }^{13}$ This multiplexed TES technology offers the possibility of producing arrays larger than the HAWC/SHARC II arrays with significantly smaller thermal loads from the readout electronics and wiring.

\section{ACKNOWLEDGMENTS}

We wish to thank M. Freund and V. Ponce for their efforts in testing and characterizing the 32 element linear arrays. The development of the PUD was initiated under the Director's Discretionary Funding program at GSFC and later developed under a grant from the NASA Office of Space Science.

\section{REFERENCES}

1. S. Moseley, C. Dowell, C. Allen, and T. Phillips, "Semiconducting pop-up bolometers for far-infrared and submillimeter astronomy," in Imaging at Radio through Submillimeter Wavelengths, J. Mangum and S. Radford, eds., ASP Conference Series 217, pp. 140-149, 2000.

2. C. D. Dowell, C. Allen, S. Babu, M. Freund, M. Gardner, J. Groseth, M. Jhabvala, A. Kovacs, D. Lis, S. Moseley, T. Phillips, R. Silverberg, G. Voellmer, and H. Yoshida, "SHARC II: a Caltech Submillimeter Observatory facility camera with 384 pixels," in Millimeter and Submillimeter Detectors for Astronomy, T. Phillips and J. Zmuidzinas, eds., Proc. SPIE 4855, p. 73, SPIE Intl. Soc. for Opt. Eng., 2002. 
3. D. Harper, C. Allen, M. Amato, T. Ames, A. Bartels, S. Casey, R. Derro, R. Evans, I. Gatley, S. Heimsath, A. Hermida, M. Jhabvala, J. H. Kastner, R. Loewenstein, S. Moseley, R. Pernic, T. Rennick, H. Rhody, D. Sanford, R. Shafer, R. Shirron, G. Voellmer, S. Wang, and J. Wirth, "HAWC: a far-infrared camera for SOFIA," in Airborne Telescope Systems, R. Melugin and H. Röser, eds., Proc. SPIE 4014, pp. 43-53, SPIE Intl. Soc. for Opt. Eng., 2000.

4. M. J. Griffin, J. J. Bock, and W. K. Gear, "Relative perfromance of filled and feedhorn-coupled focal-plane architectures," Appl. Optics 41, p. 6543, 2002.

5. S. H. Moseley, E. Wollack, and G. Hinshaw, "Limits on the efficiency of imaging systems," in Proceedings SPIE Int. Soc. Opt. Eng., J. Wolf, J. Farhoomand, and C. McCreight, eds., p. 388, SPIE Intl. Soc. for Opt. Eng., 2002.

6. G. Voellmer, C. Allen, M. Amato, A. Bartels, D. Benford, R. Derro, C. Dowell, D. Harper, S. Moseley, P. Shirron, W. Smith, and J. Staguhn, "Design and fabrictaion of two-dimensional semiconducting bolometer arrays for HAWC and SHARC-II," in Millimeter and Submillimeter Detectors for Astronomy, T. Phillips and J. Zmuidzinas, eds., 4855, p. 73, Proc. SPIE Int. Soc. Opt. Eng., 2003.

7. M. Freund, R. Silverberg, V. Ponce, C. Allen, C. Sappington, S. Moseley, D. Harper, R. Loewenstein, and C. Dowell, "Testing and performance results for the $12 \times 32$ SHARC II and HAWC bolometer arrays," in Millimeter and Submillimeter Detectors for Astronomy, T. Phillips and J. Zmuidzinas, eds., Proc. SPIE 4855, p. 73, 2002.

8. J. Beeman and E. Haller, "Far-ir calibration sources for cryogenic focal planes," in Far-IR, Submm and mm Detector Technology Workshop, J. Wolf, J. Farhoomand, and C. McCreight, eds., Proc. of NASA workshop, pp. 3-11, April 2002.

9. C. D. Dowell et al., "350 micron observations of the the Antennae Nebula," 2004. in preparation.

10. A. Kosowsky, "The Atacama Cosmology Telescope," New Astron. Rev. 47, p. 939, 2003.

11. D. J. Benford, S. H. Moseley, G. J. Stacy, R. A. Shafer, and J. G. Staguhn, "Far-infrared imaging spectroscopy with SAFIRE on SOFIA," in Airborne Telescope Systems II, R. Melugin and H. Röser, eds., 4857, p. 105B, SPIE Intl. Soc. for Opt. Eng., 2003.

12. J. G. Staguhn, D. J. Benford, K. D. Irwin, S. H. Moseley, F. Pajot, R. A. Shafer, and G. J. Stacey, "Multiplexed TES bolometers on FIBRE, SPIFI, and SAFIRE," Bulletin of the American Astronomical Society 33, p. 1525, 2001.

13. K. D. Irwin, "SQUID Multiplexers for Transition-edge Sensors," Physica C 368, pp. 203-210, 2002. 